\title{
Associação entre tempo excessivo frente à TV e índice de massa corporal em adolescentes brasileiros: uma análise de regressão quantílica da PeNSE, 2015
}

\author{
Association between excessive TV viewing time and body mass \\ index among Brazilian adolescents: quantile regression analysis \\ of the National Student Health Survey (PeNSE), 2015
}

Camilo Luis Monteiro Lourenço (https://orcid.org/0000-0003-0231-1702) ${ }^{1}$

Marina Christofoletti (https://orcid.org/0000-0002-5672-6869) ${ }^{1}$

Deborah Carvalho Malta (https://orcid.org/0000-0002-8214-5734) ${ }^{2}$

Edmar Lacerda Mendes (https://orcid.org/0000-0001-8182-8296) ${ }^{3}$

${ }^{1}$ Universidade Federal do Vale do São Francisco. Av. José de Sá Maniçoba s/n, Centro. 56304-917

Petrolina PE Brasil. camilo_lourenço@ outlook.com

${ }^{2}$ Universidade Federal de Minas Gerais. Belo Horizonte MG Brasil. ${ }^{3}$ Universidade Federal do Triângulo Mineiro. Uberaba MG Brasil.

\begin{abstract}
The study aimed to investigate whether excessive $T V$ viewing time is associated with body mass index (BMI), irrespective of physical activity (PA) and interaction with consumption of ultra-processed foods (UPFs), among Brazilian adolescents. It is a cross-sectional study with data on 13-17-year-old adolescents from the $2015 \mathrm{Na}$ tional Student Health Survey. BMI was the outcome (direct measurement of height and body mass) and exposure was excessive TV viewing time $(>2$ h/day), daily consumption of UPFs and PA. Quantile regression was used to test associations and interactions. Excessive screen time was associated with higher BMI values (e.g., 25th percentile $=0.060 \mathrm{~kg} / \mathrm{m}^{2}$ versus 95 th percentile $=0.891$ $\left.\mathrm{kg} / \mathrm{m}^{2}, p<0.001\right)$. After adjusting for PA, the association remained, however, attenuated. The inclusion of UPFs in the model potentiated the association (e.g., 25 th percentile $=0.062 \mathrm{~kg} / \mathrm{m}^{2}$ versus 95 th percentile $\left.=0.956 \mathrm{~kg} / \mathrm{m}^{2}, p<0.001\right)$. Excessive screen time was associated with BMI percentiles, irrespective of $P A$ - the absence of $P A$ and the presence of UPF consumption affect the upper BMI percentiles more. Actions to reduce excessive TV viewing time, consumption of UPFs and promoting $P A$ are essential to positively impact BMI and its reflexes on the health of Brazilian adolescents. Key words Motor activity, Industrialized foods, Nutritional status
\end{abstract}

Resumo O objetivo do estudo foi investigar se tempo excessivo frente à $T V$ está associado com indice de massa corporal (IMC), independente da atividade física $(A F)$ e da interação com consumo de alimentos ultraprocessados (AUPs), em adolescentes brasileiros. Estudo transversal com dados de adolescentes de 13-17 anos da Pesquisa Nacional de Saúde do Escolar 2015. IMC foi o desfecho (medida direta da estatura e massa corporal) e as exposições foram tempo excessivo frente à $T V(>2 \mathrm{~h} / \mathrm{dia})$, consumo diário de AUPs e AF. Regressão quantílica foi usada para testar associações e interações. Tempo excessivo frente à $T V$ foi associado a maiores valores de IMC (ex.: $25^{\circ}$ percentil $=0,060 \mathrm{~kg} / \mathrm{m}^{2}$ versus $95^{\circ}$ percentil $=0,891$ $\left.\mathrm{kg} / \mathrm{m}^{2}, p<0,001\right)$. Após ajuste para AF, a associação se manteve, porém, atenuada. A inclusão de AUPs no modelo potencializou a associação (ex.: $25^{\circ}$ percentil $=0,062 \mathrm{~kg} / \mathrm{m}^{2}$ versus $95^{\circ}$ percentil $\left.=0,956 \mathrm{~kg} / \mathrm{m}^{2}, p<0,001\right)$. Tempo excessivo frente à $T V$ foi associado a percentis do IMC, independente da AF - ausência da AF e presença do consumo de AUPs afetam mais os percentis superiores do IMC. Ações para reduzir tempo excessivo frente à $T V$, consumo de AUPs e fomentar AF, são essenciais para impactar positivamente no IMC e seus reflexos na saúde dos adolescentes brasileiros.

Palavras-chave Atividade motora, Alimentos industrializados, Estado Nutricional 


\section{Introdução}

Nas últimas décadas, a prevalência de excesso de peso na população geral tem crescido em proporções epidêmicas ${ }^{1-3}$. Estimativas nacionais ${ }^{3}$ e globais ${ }^{1}$ mostram uma assimetria crescente em direção à direita na distribuição do índice de massa corporal (IMC) das populações. Entre adolescentes brasileiros, por exemplo, passados 34 anos desde a primeira edição da Pesquisa Nacional de Despesa Familiar (1974-1975), a prevalência de excesso de peso aumentou em seis vezes no sexo masculino (de 3,7\% para $21,7 \%$ ) e em quase três vezes no sexo feminino (de $7,6 \%$ para $19,4 \%$ ) - a evolução da prevalência de obesidade em ambos os sexos repete, com menores prevalências, a tendência crescente relatada para o excesso de peso ${ }^{3}$. Essas informações são relevantes na perspectiva de saúde pública, pois adolescentes com maiores valores de IMC são aqueles com maiores riscos cardiometabólicos ${ }^{4}$ e também maior propensão ao acometimento por outras comorbidades ${ }^{5}$.

O excesso de peso possui caráter multifatorial e de grande complexidade na interação entre os possíveis fatores etiológicos ${ }^{5,6}$. O balanço energético positivo, resultado da ingestão calórica excessiva e do baixo gasto energético, figura-se como a causa mais comum para o ganho de peso na infância e adolescência ${ }^{5,6}$. Em vista disso, fatores ambientais/comportamentais como atividade física (AF), comportamentos sedentários e consumo de alimentos ultraprocessados (AUPs) têm se destacado como determinantes no atual cenário epidêmico de excesso de peso na adolescência ${ }^{7}$.

Comportamento sedentário é definido como comportamentos de baixo gasto energético $(\leq 1,5$ METs) realizados durante o tempo acordado ${ }^{8}$. O tempo excessivo frente à TV (tempo superior 2 h/dia), um tipo de comportamento sedentário de tela ${ }^{8}$, é recorrentemente desaconselhado por diretrizes internacionais ${ }^{9}$ e nacionais ${ }^{10}$. Apesar disso, esse tipo de comportamento é frequente entre adolescentes e está associado com excesso de peso ${ }^{11}$ independentemente da $\mathrm{AF}^{12,13}$. Por exemplo, tempo excessivo de TV foi $49 \%$ e $174 \%$ mais prevalentes em brasileiros com sobrepeso e obesidade $^{13}$, respectivamente. Em outro estudo ${ }^{12}$, tempo excessivo frente à TV foi associado de maneira linear com IMC. Esses resultados têm reconhecida importância epidemiológica, no entanto, usar de métodos estatísticos convencionais, como regressões binárias ${ }^{13}$ ou lineares ${ }^{12}$, pode limitar o entendimento da relação existente entre as duas variáveis. O IMC é um exemplo de desfe- cho no qual a relevância para a saúde dos adolescentes pode estar na exploração das associações nos percentis superiores da sua distribuição ${ }^{4}$, e os métodos estatísticos convencionais parecem não apresentar recursos para tratar com tais comportamentos dos dados.

A regressão quantílica, técnica estatística de regressão que modela os dados à mediana e a diferentes percentis da distribuição ${ }^{14}$ (ex.: $10^{\circ}, 2^{\circ}$, $\left.90^{\circ}, \ldots\right)$, por outro lado, é um recurso estatístico viável e robusto para fins de exploração em estudos epidemiológicos ${ }^{14,15}$. Apesar disso, seu uso permanece limitado ${ }^{14}$, especialmente em estudos de comportamento sedentário e IMC entre adolescentes ${ }^{16}$. Um estudo prévio com amostra que incluiu adolescentes (6 a 18 anos) mostrou que tempo excessivo frente à TV foi positivamente associado com IMC, independente da $\mathrm{AF}$, e que as associações foram mais fortes nos percentis superiores da distribuição do IMC. Os autores especularam que as fortes associações encontradas entre tempo excessivo frente à TV e os percentis do IMC seriam explicadas pela relação do tipo de comportamento sedentário investigado com consumo de alimentos de baixa qualidade nutricional ${ }^{16}$, mas essa possível interação não foi analisada no referido estudo.

Estudos prévios mostraram que o tempo excessivo frente à TV está relacionado com consumo de alimentos de baixa qualidade nutricional ${ }^{17,18}$. Costa et al. ${ }^{18}$, por exemplo, mostraram que aproximadamente $42 \%$ dos adolescentes brasileiros em comportamentos sedentários consumiam diariamente algum tipo de alimento com elevado grau de processamento. Os AUPs compõem um grupo alimentar derivado de formulações de uso exclusivo da indústria alimentícia, que são nutricionalmente desbalanceados e cuja produção tem por finalidade um produto pronto pra comer, palatável e de altíssimo apelo visual ${ }^{19}$ midiático. Alguns exemplos de AUPs são hambúrgueres, refrigerantes e outras bebidas açucaradas, salgadinho de pacote e biscoitos salgados $^{19}$. Além disso, consumo de AUPs também está associado a chance aumentada de obesida$\mathrm{de}^{20}$ e à síndrome metabólica em adolescentes ${ }^{21}$. Apesar disso, ainda não é clara a interação entre tempo excessivo frente à TV e consumo de AUPs na relação com o IMC de adolescentes ${ }^{11,16,17}$.

Considerando as lacunas de pesquisa envolvendo a relação entre comportamento sedentário, $\mathrm{AF}$, consumo de alimentos de baixa qualidade nutricional e o IMC, o objetivo do estudo foi investigar se tempo excessivo frente à TV está associado com IMC, independentemente da AF e 
interação com consumo de AUPs, em adolescentes brasileiros.

\section{Métodos}

\section{Desenho do estudo}

Estudo transversal, com uso de dados da terceira edição da PeNSE conduzida entre abril e setembro de 2015 com amostra de representatividade nacional dos escolares brasileiros do $6^{\circ}$ ano do Ensino Fundamental à $3^{\text {a }}$ série do Ensino Médio (nomeada Amostra 2 da PeNSE 2015)22. A amostra incluiu alunos de escolas privadas e públicas das zonas urbana e rural, com ao menos 15 estudantes regularmente matriculados. $\mathrm{Na}$ PeNSE 2015, as cinco grandes regiões do país (Norte, Nordeste, Centro-Oeste, Sudeste e Sul) foram consideradas como os estratos geográficos. A seleção amostral de estudantes foi realizada seguindo esquema de amostragem por conglomerados. Foram selecionadas escolas em cada uma das grandes regiões e, nessas escolas, foram selecionadas as turmas, nas quais todos os escolares foram elegíveis para participar do inquérito e foram convidados a responder ao questionário da pesquisa. O levantamento de informações da Amostra 2 da PeNSE 2015 foi realizado com adolescentes de diferentes idades, contudo, para garantir a representatividade da amostra de escolares de 13 a 17 anos e possibilitar a comparabilidade dos dados com indicadores internacionais, especialmente aqueles oriundos do Global School-based Student Health Survey da Organização Mundial da Saúde ${ }^{23}$, foram filtrados no banco de dados somente adolescentes de 13 a 17 anos de idade $^{22}$. Ainda, a Amostra 2 da PeNSE 2015 fornece medida objetiva da massa corporal e estatura para cálculo do IMC, conforme descrito a seguir.

O dimensionamento da amostra levou em consideração erro amostral máximo aproximado de $3 \%$, em valor absoluto, proporção da ordem de 50\% (para a maximizar as variâncias dos estimadores amostrais) ao nível de confiança de 95\% e efeito médio do plano amostra, no primeiro estágio, da ordem de 3. Mais detalhes sobre as características metodológicas e de amostragem da PeNSE 2015 podem ser acessadas em publicação prévia $^{24}$. A PeNSE 2015 foi aprovada pela Comissão Nacional de Ética em Pesquisa do Conselho Nacional de Saúde.

\section{Variáveis}

Estimativas de tempo excessivo frente à TV ( 2 h/dia) e do tempo de Atividade Física

Os adolescentes responderam de maneira autônoma a questões sobre tempo de TV e de atividade física (AF) por meio de smartphones. Tempo de TV foi estimado pela seguinte questão "Em um dia de semana comum, quantas horas por dia você assiste a TV?” - essa questão não contabiliza sábado, domingo e feriado ${ }^{22}$. Considerou-se quantidade de tempo superior a $2 \mathrm{~h} / \mathrm{dia}$ como tempo excessivo frente à TV, baseado nas diretrizes da Sociedade Brasileira de Pediatria ${ }^{10} \mathrm{e}$ da American Academy of Pediatrics ${ }^{9}$.

AF foi estimada pelo produto entre o número de dias e o tempo médio gasto em AF, nos sete dias anteriores à pesquisa, considerando os seguintes domínios: ir e voltar da escola a pé ou de bicicleta, aulas de educação física e outras atividades extraescolares. Em seguida, a variável foi categorizada em suficientemente ativos $(\geq 300$ $\mathrm{min} /$ semana $)$ e insuficientemente ativo $(<300$ min/semana), baseado no Physical Activity Guidelines Advisory Committee Report ${ }^{25}$.

\section{Covariáveis}

Covariáveis foram selecionadas considerando suas relações com as exposições e desfechos desse estudo ${ }^{5,11,12,16,26,27}$. Assim, foram consideradas covariáveis relacionadas à escola ${ }^{26,27}$ : tipo de administração (pública e privada) e localização (urbana e rural), séries ( $6^{\circ}$ ao $9^{\circ}$ ano do ensino fundamental e $1^{\circ}$ ao $3^{\circ}$ ano do ensino médio), turnos (manhã, intermediário, tarde, noite e integral) e regime escolar (ensino integral e internato). A variável referente às regiões do país (regiões Norte, Nordeste, Centro-Oeste, Sudeste e Sul) foi inserida como covariável para ajustar para possíveis discrepâncias regionais. Idade (13 a 17 anos) $)^{5,12,26}$, cor da pele (branca, preta, parda e amarela/indígena) ${ }^{16}$ e sexo (masculino e feminino $)^{5,26}$ também foram usadas como covariáveis.

Consumo diário de ao menos um grupo de AUPs foi considerado covariável e obtida por meio do item: "Nos últimos 7 dias, em quantos dias você comeu...", para os alimentos/grupos de alimentos a seguir: refrigerantes, industrializados/ultraprocessados (salgados, como hambúrguer, presunto, mortadela, salame, linguiça, salsicha, macarrão instantâneo, salgadinho de pacote, biscoitos salgados) e guloseimas (doces, balas, chocolates, chicletes, bombons ou pirulitos). Consumo diário foi considerado quando da resposta afirmativa ao consumo de ao menos 
um dos grupos de alimentos em todos os dias da semana. A denominação de AUPs foi baseado na classificação NOVA ${ }^{19}$.

Medidas antropométricas e cálculo do IMC Apenas os participantes da Amostra 2 da PeNSE 2015 tiveram medidas antropométricas obtidas por medida direta, realizadas por técnicos do Instituto Brasileiro de Geografia e Estatística $^{22}$. Medidas antropométricas foram realizadas após o adolescente finalizar o questionário, fora da sala de aula, em local adequado. Massa corporal foi registrada em quilogramas e obtida por meio de balança eletrônica portátil, considerando-se uma casa decimal no visor. A estatura foi registrada em centímetros e obtida por meio de estadiômetro portátil, fixado em parede, considerando-se uma casa decimal. $\mathrm{Na}$ impossibilidade de mensuração, recusa ou valores discrepantes, massa corporal e estatura foram imputados, considerando a idade e o sexo, por meio de conjunto de rotinas computacionais do pacote Statistical Analysis System (SAS), integrada à Metodologia de Crítica e Imputação de Dados Quantitativos (CIDAQ) ${ }^{22}$. O IMC foi obtido pela massa corporal dividida pela estatura ao quadrado $\left(\mathrm{kg} / \mathrm{m}^{2}\right)$.

\section{Métodos estatísticos}

Estatísticas descritivas são apresentadas como medianas (amplitude interquartil), frequências absolutas e percentuais. Testes de Kolmogorov -Smirnov (K-S) revelaram distribuição não-paramétrica para IMC e AF em ambos os sexos. Diferenças entre os sexos foram acessadas pelo teste Qui-quadrado para nível de AF e consumo diário de AUPs e teste U de Mann-Whitney para IMC.

Regressão quantílica foi utilizada para testar associação entre tempo excessivo frente à TV (>2 h/dia) e IMC $\left(\mathrm{kg} / \mathrm{m}^{2}\right)$. Modelos de regressão quantílica são recentemente apresentados como importante estratégia estatística nesse campo de investigação ${ }^{16}$. Essa proposta visa contemplar a melhor interpretação dos dados inseridos em modelos de regressão, os quais oferecem inferências para diferentes percentis, não apenas da mediana, respeitando ainda três importantes conceitos estatísticos: regressão, robustez e teoria dos valores extremos $^{15}$. O coeficiente de regressão quantílica quantifica quanto um determinado percentil da distribuição da variável dependente é alterado pelo incremento da variável preditora ${ }^{14}$.

IMC foi inserido como variável dependente nos modelos construídos (coeficientes são, portanto, interpretados como incremento ou redu- ção em $\mathrm{kg} / \mathrm{m}^{2}$ ). Para atender ao objetivo deste estudo, três modelos de regressão quantílica foram elaborados e ajustados de modo sucessivo. $\mathrm{O}$ Modelo 1 testou associação entre tempo excessivo frente à TV e IMC, ajustado para covariáveis (escola, estrato geográfico e idade, cor da pele e sexo). Em seguida, foram adicionados AF ao Modelo 2 e consumo diário de AUPs ao Modelo 3. Termos de interação entre tempo excessivo frente à TV ( $\geq 2 \mathrm{~h} / \mathrm{dia})^{\star} \mathrm{AF}$ (suficientemente ativo) e tempo excessivo frente à $\mathrm{TV}^{\star} \mathrm{AUPs}$ (consumo diário) foram criados para indicar diferença na magnitude do IMC com inclusão desses termos nos modelos de regressão quantílica. Desse modo, os coeficientes das interações representam a diferença na mudança do valor do IMC em kg/ $\mathrm{m}^{2}$ no $\mathrm{n}^{\mathrm{o}}$ percentil comparada à mudança quando a variável de interação é mantida no seu nível de referência; ou seja, tempo excessivo frente à $\mathrm{TV}^{\star} \mathrm{AF}$ representa a interação do tempo de TV (acima de $2 \mathrm{~h} / \mathrm{dia}$ ) com AF (suficientemente ativo); e tempo excessivo frente à $\mathrm{TV}^{\star} \mathrm{AUPs}$ representa a interação do tempo de TV (acima de 2 h/dia) com AUPs (consumo diário).Em todos os modelos, o método enter foi usado para inclusão das covariáveis (escola, estrato geográfico e idade, cor da pele e sexo).

Foram testadas associações entre tempo excessivo frente à TV vs. quartis do $\operatorname{IMC}\left(25^{\circ}, 50^{\circ}\right.$ e $\left.75^{\circ}\right)$, além de dois percentis mais altos $\left(90^{\circ} \mathrm{e}\right.$ 950) da distribuição. Foi adotada largura de banda Bofinger, com exclusão de casos omissos, tolerância de singularidade de $10^{-12}$, convergência de $10^{-6}$ e erros padrões estimados de 2.000 amostras bootstrap nas regressões quantílicas. Para fins comparativos, foram realizadas análises de regressão linear múltipla com as mesmas variáveis (dependente e covariáveis - tempo excessivo frente à TV, AF e consumo de AUPs foram inseridos apenas como variáveis de ajuste) para apresentação dos coeficientes de regressões lineares $\left(\beta_{\text {linear }}\right)$. As análises incorporaram pesos amostrais, para considerar o plano amostral complexo $^{22}$, e p $<0,01$ (IC99\%) foi considerado como significância estatística. Todas as análises estatísticas foram realizadas no pacote SPSS 26.

\section{Resultados}

\section{Descrição da amostra}

Foram analisados dados válidos de 10.926 escolares de 13 a 17 anos de idade. As características dos participantes, para toda a amostra 
e estratificada por sexo, foram apresentadas na Tabela 1. A maioria das escolas eram públicas $(75,8 \%)$ e pouco mais da metade dos escolares foram do sexo masculino (50,5\%), com maior proporção de adolescentes autorreferindo-se pardos $(43,6 \%)$. A maior parte da amostra passava tempo excessivo frente à TV $(>2 \mathrm{~h} / \mathrm{dia})$ $(52,2 \%)$ e foi considerada insuficientemente ativa $(67,6 \%)$. O consumo diário de AUPs foi reportado por $40,0 \%$ dos escolares. Adolescentes do sexo feminino apresentaram maior tempo excessivo frente à TV $(>2 \mathrm{~h} /$ dia $)(\mathrm{p}<0,001)$, nível insuficiente de $\mathrm{AF}(\mathrm{p}<0,001)$ e consumo diário de AUPs $(p<0,001)$ em relação ao sexo masculino (Tabela 1).

Em toda a amostra, os escolares apresentaram IMC mediano de $20,5(4,6) \mathrm{kg} / \mathrm{m}^{2}$. O sexo masculino apresentou IMC de $20,2(4,5) \mathrm{kg} / \mathrm{m}^{2}$ e o sexo feminino de $20,8(4,7) \mathrm{kg} / \mathrm{m}^{2}$, com diferença entre os sexos $(\mathrm{p}<0,001)$. A mediana do tempo de AF foi 180 (285) $\mathrm{min} / \mathrm{semana}$. O sexo masculino apresentou 235,0 (325) $\mathrm{min} / \mathrm{semana}$ de mediana do tempo de AF e o sexo feminino 135,0 (225) $\mathrm{min} / \mathrm{semana}$.

\section{Regressões quantílicas e interações}

Tempo excessivo frente à TV $(>2 \mathrm{~h} /$ dia $)$ foi associado a maiores valores de IMC e, em todos os modelos, os gradientes das associações foram ainda mais fortes nos percentis superiores da distribuição do IMC (Tabela 2). Por exemplo, no $25^{\circ}$ percentil do Modelo 1 , tempo excessivo frente à TV foi associado com IMC $0,060 \mathrm{~kg} / \mathrm{m}^{2}$ maior; por outro lado, nos percentis $90^{\circ}$ e no $95^{\circ}$, tempo excessivo frente à TV foi associado com IMC 0,576 e $0,891 \mathrm{~kg} / \mathrm{m}^{2}$ maior, respectivamente (Modelo 1).

Independentemente da AF (Modelo 2, Tabela 2), tempo excessivo frente à TV foi associado a maiores valores de IMC, mas com gradientes reduzidos nos percentis inferiores da distribuição. $\mathrm{O}$ ajuste para $\mathrm{AF}$ atenuou a força da associação entre tempo excessivo frente à TV e IMC apenas nos percentis $25^{\circ}$ e $50^{\circ}$ da distribuição, conforme termos de interação produto cruzado. No percentil $25^{\circ}$, por exemplo, o termo de interação mostra que tempo excessivo frente à TV foi associado com IMC $0,280 \mathrm{~kg} / \mathrm{m}^{2}$ menor entre os adolescentes suficientemente ativos.

A inclusão do consumo diário de AUPs (Modelo 3, Tabela 2) aumentou a força de associação entre tempo excessivo frente à TV e IMC. Por exemplo, no $25^{\circ}$ percentil do Modelo 3, tempo excessivo frente à TV foi associado com IMC $0,062 \mathrm{~kg} / \mathrm{m}^{2}$ maior; por outro lado, nos percentis $90^{\circ}$ e no $95^{\circ}$, tempo excessivo frente à TV foi associado com IMC 0,688 e $0,956 \mathrm{~kg} / \mathrm{m}^{2}$ maior, res-

Tabela 1. Características dos escolares participantes da Pesquisa Nacional de Saúde do Adolescente (PeNSE). Brasil, 2015 ( $\mathrm{N}=10.926)$.

\begin{tabular}{|c|c|c|c|c|c|c|c|}
\hline \multirow{2}{*}{ Variáveis } & \multicolumn{2}{|c|}{ Todos } & \multicolumn{2}{|c|}{ Masculino } & \multicolumn{2}{|c|}{ Feminino } & \multirow{2}{*}{ p } \\
\hline & $\mathbf{n}$ & $\%$ & $\mathbf{n}$ & $\%$ & $\mathbf{n}$ & $\%$ & \\
\hline Cor da pele ${ }^{a}$ & & & & & & & $<0,001$ \\
\hline Branca & 4.300 & 36,2 & 2.335 & 36,9 & 2.065 & 35,5 & \\
\hline Preta & 1.277 & 13,2 & 759 & 16 & 518 & 10,4 & \\
\hline Parda & 4.560 & 43,6 & 2.158 & 40,3 & 2.402 & 46,9 & \\
\hline Amarela/Indígena & 781 & 7,0 & 363 & 6,8 & 418 & 7,2 & \\
\hline Tipo de escola & & & & & & & $<0,001$ \\
\hline Pública & 8.287 & 75,8 & 4.188 & 87,5 & 4.099 & 86,6 & \\
\hline Privada & 2.639 & 24,2 & 1.334 & 12,5 & 1.305 & 13,4 & \\
\hline Tempo frente à $\mathrm{TV}^{\mathrm{b}}$ & & & & & & & $<0,001$ \\
\hline $0-2 \mathrm{~h} / \mathrm{dia}$ & 5.210 & 47,8 & 48,2 & 2.719 & 44,1 & 2.491 & \\
\hline Acima de 2 h/dia (excessivo) & 5.686 & 52,2 & 51,8 & 2.780 & 55,9 & 2.906 & \\
\hline Atividade Física $^{c}$ & & & & & & & $<0,001$ \\
\hline Suficientemente ativo & 3.533 & 32,4 & 2.270 & 41,1 & 1.263 & 22,1 & \\
\hline Insuficientemente ativo & 7.380 & 67,6 & 3.243 & 58,9 & 4.137 & 77,1 & \\
\hline Consumo diário de AUPs ${ }^{\mathrm{d}}$ & & & & & & & $<0,001$ \\
\hline Sim & 4.237 & 40,0 & 1.953 & 36,2 & 2.284 & 43,7 & \\
\hline Não & 6.640 & 60,0 & 3.537 & 63,8 & 3.103 & 56,3 & \\
\hline
\end{tabular}

AUPs: alimentos ultraprocessados. Valores ausentes: ${ }^{a} n=8 ;{ }^{b} n=30 ;{ }^{c} n=13 ;{ }^{d} n=49$. 
Tabela 2. Modelos de regressão quantílica da associação entre tempo frente à TV e percentis do IMC em participantes da Pesquisa Nacional de Saúde do Adolescente (PeNSE). Brasil, 2015 (N=10.926).

\begin{tabular}{|c|c|c|c|c|c|}
\hline \multirow{2}{*}{$\begin{array}{c}\begin{array}{c}\text { Tempo excessivo } \\
\text { frente à de TV }\end{array} \\
\text { Ref. } 0-2 \mathrm{~h} / \mathrm{d} \\
\end{array}$} & \multicolumn{5}{|c|}{ Percentis do IMC e IC99\% } \\
\hline & $25^{\circ}($ IC $99 \%)$ & $50^{\circ}(\mathrm{IC} 99 \%)$ & $75^{\circ}($ IC $99 \%)$ & $90^{\circ}($ IC $99 \%)$ & $95^{\circ}($ IC99\%) \\
\hline \multicolumn{6}{|l|}{ Modelo 1} \\
\hline \multicolumn{6}{|l|}{$>2 \mathrm{~h} / \mathrm{d}$} \\
\hline$\beta$ & $0,06(0,05 ; 0,06)$ & $0,09(0,09 ; 0,10)$ & $0,37(0,36 ; 0,38)$ & $0,58(0,56 ; 0,59)$ & $0,89(0,87 ; 0,91)$ \\
\hline $\mathrm{EP}$ & 0,002 & 0,002 & 0,004 & 0,006 & 0,009 \\
\hline $\mathrm{p}$ & $<0,001$ & $<0,001$ & $<0,001$ & $<0,001$ & $<0,001$ \\
\hline \multicolumn{6}{|l|}{ Modelo 2} \\
\hline \multicolumn{6}{|l|}{$>2 \mathrm{~h} / \mathrm{d}$} \\
\hline$\beta$ & $0,03(0,03 ; 0,04)$ & $0,09(0,08 ; 0,09)$ & $0,38(0,37 ; 0,39)$ & $0,60(0,58 ; 0,61)$ & $0,87(0,85 ; 0,89)$ \\
\hline $\mathrm{EP}$ & 0,002 & 0,002 & 0,004 & 0,007 & 0,008 \\
\hline $\mathrm{p}$ & $<0,001$ & $<0,001$ & $<0,001$ & $<0,001$ & $<0,001$ \\
\hline \multicolumn{6}{|l|}{$\begin{array}{l}\text { Tempo excessivo } \\
\text { frente à TV vs. } \mathrm{AF}^{\#}\end{array}$} \\
\hline$\beta$ & $-0,28(-0,30 ;-0,27)$ & $-0,06(-0,08 ;-0,05)$ & $0,36(0,35 ; 0,38)$ & $0,38(0,350 ; 0,41)$ & $0,56(0,52 ; 0,60)$ \\
\hline $\mathrm{EP}$ & 0,003 & 0,004 & 0,006 & 0,011 & 0,015 \\
\hline $\mathrm{p}$ & $<0,001$ & $<0,001$ & $<0,001$ & $<0,001$ & $<0,001$ \\
\hline \multicolumn{6}{|l|}{ Modelo 3} \\
\hline \multicolumn{6}{|l|}{$>2 \mathrm{~h} / \mathrm{d}$} \\
\hline$\beta$ & $0,06(0,06 ; 0,07)$ & $0,16(0,15 ; 0,16)$ & $0,39(0,38 ; 0,40)$ & $0,69(0,67 ; 0,70)$ & $0,96(0,93 ; 0,98)$ \\
\hline $\mathrm{EP}$ & 0,002 & 0,002 & 0,004 & 0,006 & 0,008 \\
\hline $\mathrm{p}$ & $<0,001$ & $<0,001$ & $<0,001$ & $<0,001$ & $<0,001$ \\
\hline \multicolumn{6}{|l|}{$\begin{array}{l}\text { Tempo excessivo } \\
\text { frente à TV vs. } \\
\text { AUPs\# }\end{array}$} \\
\hline$\beta$ & $0,10(0,10 ; 0,11)$ & $0,31(0,30 ; 0,32)$ & $0,44(0,42 ; 0,45)$ & $0,86(0,83 ; 0,89)$ & $1,58(1,54 ; 1,61)$ \\
\hline EP & 0,003 & 0,004 & 0,006 & 0,01 & 0,014 \\
\hline $\mathrm{p}$ & $<0,001$ & $<0,001$ & $<0,001$ & $<0,001$ & $<0,001$ \\
\hline
\end{tabular}

IMC: índice de massa corporal $\left(\mathrm{kg} / \mathrm{m}^{2}\right) ; 25^{\circ},(\ldots), 95^{\circ}$ : percentis de distribuição do IMC; IC99\%: intervalo de confiança de 99\%; $\beta$ : coeficiente beta; EP: erro padrão (estimado de 2.000 amostras bootstrap); AF: atividade física; AUPs: alimentos ultraprocessados. Modelo 1: ajuste para variáveis da escola [tipo (pública e privada), localização (urbana e rural), séries, turnos e o regime escolar (ensino integral e internato)], estrato geográfico, idade, cor da pele e sexo; Modelo 2: ajuste para o Modelo 1 + AF; Modelo 3: ajuste para Modelos 1 e 2 + AUPs. *: coeficientes das interações representam a diferença na mudança do valor do $\mathrm{IMC} \mathrm{em} \mathrm{kg} / \mathrm{m}^{2}$ no $\mathrm{n}^{\circ}$ percentil comparada à mudança quando a variável de interação é mantida no seu nível de referência: Tempo excessivo frente à de TV ${ }^{\star} \mathrm{AF}$ : representa a interação do tempo de TV (acima de $2 \mathrm{~h} / \mathrm{dia}$ ) com AF (suficientemente ativo); Tempo excessivo frente à de $\mathrm{TV}^{\star} \mathrm{AUPs}$ : representa a interação do tempo de TV (acima de $2 \mathrm{~h} / \mathrm{dia}$ ) com AUPs (consumo diário).

Fonte: Elaborado pelos autores.

pectivamente. Todos os termos de interação produto cruzado entre tempo excessivo frente à TV e AUPs mostram incremento na associação com IMC (Modelo 3, Tabela 2). No percentil mais alto (95), por exemplo, o termo de interação mostra que tempo excessivo frente à TV foi associado com IMC $1,578 \mathrm{~kg} / \mathrm{m}^{2}$ maior entre escolares com consumo diário de AUPs.

As representações visuais das associações entre tempo excessivo frente à TV $(>2 \mathrm{~h} /$ dia $)$ e distribuição dos percentis do IMC foram apresentadas na Figura 1. Conforme apresentado na Tabela
2, os resultados mostrados na Figura 1 indicam aumento da associação entre tempo excessivo frente à TV e IMC à medida em que se ajustam os modelos (Modelo $3>$ Modelo $2>$ Modelo 1). Ainda, percebe-se a mais valia da regressão quantílica sobre a regressão linear quando observado o comportamento da associação nas Figuras 1A, 1B e 1C. Para cada uma das figuras, as estimativas de IMC via regressão quantílica mostram o comportamento da associação entre tempo excessivo frente à TV e os diferentes percentis da distribuição do IMC, associando-se não apenas à 
mediana $\left(50^{\circ}\right.$ percentil), ou à média (Modelo 1 : $\beta_{\text {linear }}=0,269(0,263 ; 0,276), p<0,001$; Modelo 2: $\beta_{\text {linear }}=0,266(0,260 ; 0,272), \mathrm{p}<0,001$; Modelo 3: $\left.\beta_{\text {linear }}=0,294(0,288 ; 0,301) \mathrm{p}<0,001\right)$, mas também a percentil inferior $\left(25^{\circ}\right)$ e superiores $\left(75^{\circ}\right.$, $90^{\circ}$ e $95^{\circ}$ ).

\section{Discussão}

Os principais resultados do presente estudo sugerem associação, por meio de análises de regressão quantílica, entre tempo excessivo frente à TV e maiores valores de IMC, independente da AF, em adolescentes brasileiros. Os resultados mostraram que a força dessa associação aumentou em direção aos percentis superiores do IMC. Adicionalmente, os termos de interação mostraram que AF parece atenuar, mas não anular, a associação entre tempo excessivo frente à TV e IMC apenas em percentis inferiores. Em contrapartida, a interação do tempo excessivo frente à TV e consumo diário de AUPs parece incrementar o gradiente da associação entretempo excessivo frente à TV e IMC, com efeitos mais expressivos nos percentis superiores da distribuição do desfecho.

Este estudo com dados de escolares de todo o Brasil identificou um cenário preocupante a respeito da prática de $\mathrm{AF}$, tempo excessivo frente à TV e consumo diário de AUPs. A evolução temporal das prevalências de inadequações a valores do espectro saudável do IMC, para refletir o estado nutricional, tem se revelado alarmante entre adolescentes do Brasil $3,28,29$. Entre 1974-1975 e 2008-2009, a prevalência de sobrepeso entre adolescentes do sexo masculino evoluiu de 3,7\% para $21,7 \%$, enquanto, no sexo feminino, o crescimento foi de $7,6 \%$ para $19,4 \%$. Nesse mesmo período, a prevalência de obesidade aumentou em 15 vezes no sexo masculino (de $0,4 \%$ para $5,9 \%$ ) e seis vezes no sexo feminino (de $0,7 \%$ para $4,0 \%)^{3}$. Essa tendência de crescimento da prevalência de sobrepeso é reforçada por outra pesquisa nacional com adolescentes do sexo masculino conduzida entre os anos de 1980 e $2005^{29}$. A estimativa mais recente entre adolescentes escolares brasileiros, de ambos os sexos, aponta para prevalência de $18 \%$ de sobrepeso e $9 \%$ de obesidade ${ }^{28}$. Crianças e adolescentes identificados nos percentis mais elevados da distribuição do IMC, a exemplo do $95^{\circ}$ percentil, são mais propensas a fatores de risco cardiometabólicos como pressão arterial elevada e valores de HDL-C, triacilgliceróis e glicose de jejum alterados ${ }^{4}$. Além disso, o excesso de peso transita da infância para a adolescên-

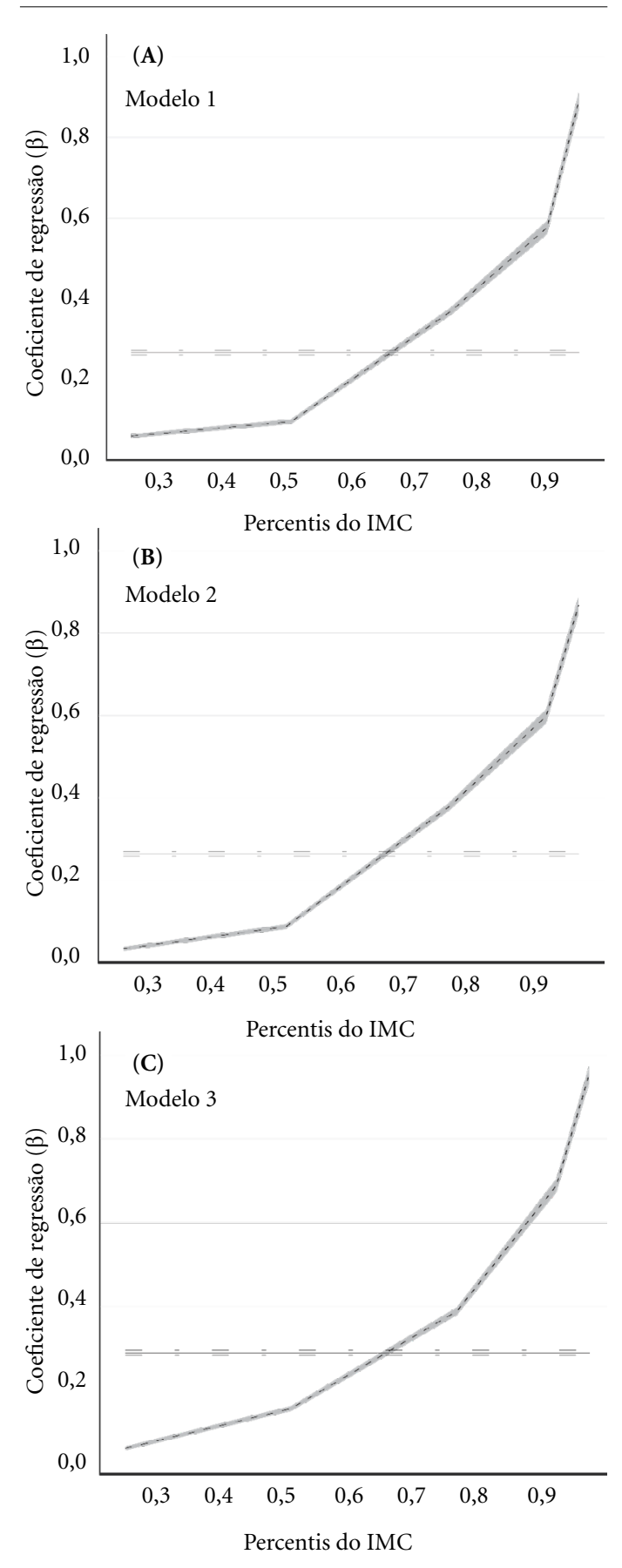

Figura 1. Associação entre tempo excessivo frente à TV e percentis do IMC por modelos de regressão quantílica em participantes da Pesquisa Nacional de Saúde do Adolescente (PeNSE). Brasil, 2015 ( $\mathrm{N}=10.926)$.

Fonte: Elaborado pelos autores.

cia $^{30}$ perdurando para a vida adulta ${ }^{5}$, sobretudo na obesidade grave, podendo elevar a chance da 
ocorrência de problemas de saúde não apenas na adolescência, como também na idade adulta.

Tempo excessivo frente à TV foi associado com IMC em todos os percentis investigados, mostrando incremento na força de associação desde o menor $\left(25^{\circ}\right)$ até o maior $\left(95^{\circ}\right)$ percentil, mesmo quando realizado ajuste para a AF. Similarmente, um estudo prévio ${ }^{16} \mathrm{com}$ amostra que incluiu adolescentes (6 a 18 anos) mostrou que tempo excessivo frente à TV ( $3 \mathrm{a} 4 \mathrm{~h} / \mathrm{dia})$ foi associado com valores aumentados de IMC, independentemente da AF, desde o menor $\left(\beta=0,008 ; 5^{\circ}\right.$ percentil) até o mais alto percentil $\left(\beta=0,40 ; 95^{\circ}\right.$ percentil) - o modelo bruto e interações entre $\mathrm{AF}$ e o tempo excessivo frente à TV não foram fornecidos. Apesar das discrepâncias metodológicas entre o presente estudo e o estudo acima mencionado ${ }^{16}$, sobretudo na definição de tempo excessivo frente à $\mathrm{TV}$, a regressão quantílica aponta que o comportamento sedentário de telas se associa de maneira distinta aos diferentes estratos do IMC. Adicionalmente, o presente estudo avançou em relação aosprévios ${ }^{12,13} \mathrm{em}$ revelar que a $\mathrm{AF}$ atenua associação entre tempo excessivo frente à TV e percentis inferiores do IMC. Métodos tradicionais de regressão empregados ${ }^{12,13}$ não permitem acessar informações alcançadas por meio do modelo de regressão quantílica.

Diante da relação inversa entre AF e IMC estabelecida na literatura ${ }^{7}$ e do delineamento de natureza transversal do presente estudo, seria plausível pressupor que as associações identificadas entre os adolescentes nos percentis inferiores do IMC apresentassem algum "efeito protetor" da prática de AF. Isso pôde ser identificado nos resultados do presente estudo, haja vista os coeficientes de regressão e da interação mostrados no Modelo 2 para adolescentes do $25^{\circ}$ percentil e da mediana do IMC. Por outro lado, é preocupante notar que adolescentes nos percentis superiores da distribuição do IMC podem não se "beneficiar" do possível efeito protetor da AF, como indicado pelos coeficientes de regressão e de interação do Modelo 2, a partir do $75^{\circ}$ percentil. Ainda, o termo de interação entre AF e tempo excessivo frente à TV foi construído sob a premissa de que adolescentes com tempo frente à TV superior a duas horas por dia estão expostos a comportamento sedentário excessivo, ${ }^{9,10}$. Entretanto, em estudo de coorte, Hancox et al. ${ }^{31}$ mostraram que o tempo gasto frente à TV inferior a uma hora por dia é preditor de alta prevalência de fatores de risco como sobrepeso, colesterol alto, fumo e baixa aptidão física aos 26 anos, mesmo ajustado para fatores de confusão. Nesse sentido, tomados em conjunto, o resultado apresentado neste estudo e $\mathrm{o}$ indicativo de que tempo excessivo frente à TV menor que o ponto de corte de duas horas se relaciona com obesidade, como apontado por Hancox et al. ${ }^{31}$, fica ainda mais evidente que os efeitos deletérios do comportamento de assistir TV pode se dar até mesmo com tempo inferior a uma hora de exposição em adolescentes. De fato, importantes sociedades científicas para a saúde da criança e do adolescente, tais como a Sociedade Brasileira de Pediatria ${ }^{10}$ e a American Academy of Pediatri$c s^{9}$, demonstraram preocupação quanto ao tempo gasto em exposição passiva frente a tela. Em suas recomendações, ambas indicam limitar esse tempo a não mais que uma hora por dia para as crianças, e não para os adolescentes. Para melhor esclarecer a suposição acima formulada, estudos futuros poderiam explorar a relação entre as diferentes quantidades de tempo passivo frente à tela e os percentis do IMC em adolescentes.

O presente estudo sugere que a interação entre tempo excessivo frente à TV e consumo diário de ao menos um grupo de AUPs aumentou a força de associação entre tempo excessivo frente à TV e IMC, especialmente nos percentis superiores da distribuição do IMC. Pesquisa anterior com dados da PeNSE 2015 (Amostra 1) mostrou que maior tempo em comportamento sedentário esteve associado com consumo diário de AUPs entre adolescentes brasileiros ${ }^{18}$. De fato, em estudos com adolescentes, o comportamento sedentário se mostra frequentemente associado a consumo de AUPs como fast foods, bebidas açucaradas e outros lanches de alta densidade energética ${ }^{17}$. Cabe ressaltar que AUPs constituem um grupo de alimentos derivados de formulações feitas principalmente ou inteiramente por substâncias de uso exclusivos das indústrias - não mantendo qualquer similaridade com os alimentos minimamente processados ou os naturais $^{19}$. Além disso, os AUPs são nutricionalmente desbalanceados, contendo quantidades elevadas de sódio, gorduras e açúcares, e cujo objetivo comum é obter um produto final cômodo (pronto pra consumir e durável), atraente (altamente palatável), lucrativo (baixo custo dos ingredientes) e comercializado e anunciado agressivamente ${ }^{19}$. A publicidade de gêneros alimentícios e bebidas é a terceira maior categoria entre anúncios na TV aberta brasileira e, dentro dessa categoria, os AUPs são responsáveis por mais de $60 \%$ dos anúncios $^{32}$. Adicionalmente, a participação percentual desse grupo alimentar tem se mostrado crescente nas residências das famílias brasileiras $^{33}$, o que pode impactar no consumo diário 
pelos adolescentes. Desse modo, o consumo diário de AUPs pode ser potencializado por indução (alta exposição à mídia televisiva ${ }^{32}$ e por oferta (crescente disponibilidade na dieta das famílias brasileiras) $)^{33}$.

Os achados do presente estudo reforçam a necessidade de redução do tempo excessivo frente à TV e do consumo de AUPs, concomitantemente ao aumento da AF no enfrentamento à obesidade. $\mathrm{O}$ aumento indesejado do peso corporal e, consequentemente, do IMC, possui caráter multifatorial ${ }^{5,6}$ apresentando uma complexa interação entre os fatores etiológicos. Conforme postulado por Pereira-Lancha et al. ${ }^{6}$, diversos são os fatores indutores da obesidade, dentre os quais o balanço energético positivo (armazenamento energético superando o gasto calórico), a dieta hiperlipídica (atuando sobre os níveis plasmáticos de leptina, que também modula a secreção de grelina, prejudicando, por fim, o balanço energético), as alterações hormonais no controle do apetite (inclui sinais hormonais do trato gastrointestinal com efeitos negativos (ex.: peptídeo 1 tipo glucagon, colecistocinina, polipeptídeo pancreático, peptídeo YY, oximodulina) e positivos (grelina) para o balanço energético), a resistência insulínica e o metabolismo dos aminoácidos têm papel de destaque, os quais interagem ou não com fatores genéticos. Pereira-Lancha et al. ${ }^{6}$ argumentam ainda que o rápido aumento na prevalência do excesso de peso, ocorrido em um curto espaço de tempo, não poderia ser creditado com exclusividade a fatores genéticos, o que reforça a tese da carga dos fatores ambientais/comportamentais. Alinhado a isso, Kumar e Kelly ${ }^{5}$ enfatizaram que os fatores ambientais/ comportamentais têm relevante parcela da carga etiológica no desenvolvimento do excesso de peso entre a população de jovens. De acordo com os autores ${ }^{5}$, fatores cuja ocorrência é concomitante e advindos das alterações do ambiente/ comportamento, ou seja, o elevado consumo calórico, a redução da prática de atividade física e o aumento nas oportunidades de permanecer em comportamentos sedentários são os responsáveis pelo atual "ambiente obesogênico". De fato, fatores ambientais/comportamentais de risco para a obesidade tendem a coexistir e a se agrupar entre os adolescentes ${ }^{34,35}$. Logo, a investigação da ocorrência desses fatores na população mais exposta, como a que o presente estudo identificou, pode contribuir para que medidas de saúde pública se concentrem na proposição de ações para a reversão do quadro epidemiológico de excesso de peso entre adolescentes no Brasil ${ }^{3,28,29}$. Além disso, as minorias populacionais representam valores expressivos destinados ao cuidado em saúde ${ }^{36,37}$. Por exemplo, estima-se que os gastos para o sistema público de saúde de uma criança com obesidade seja o dobro em comparação ao de uma criança não obesa ${ }^{36}$, podendo ser ainda mais oneroso aos serviços públicos de saúde, se consideradas as comorbidades relacionadas à obesidade ${ }^{37}$.

$\mathrm{O}$ estudo possui algumas limitações, mesmo ao considerar-se o maior estudo de monitoramento de saúde de adolescentes escolares do Brasil $^{22,24}$. As variáveis comportamentais foram respondidas por meio do autorrelato, necessitando de uma compreensão reflexiva sobre a relação de espaço-tempo. Por conter autorrelato de apenas um comportamento sedentário, acredita-se que sua duração possa estar subestimada, o que sugere cautela na interpretação dos dados. Este estudo incluiu AF realizadas pelos adolescentes nos contextos do deslocamento para casa/escola, nas aulas de Educação Física e extraescolares como esportes, dança, ginástica, musculação e lutas. Entretanto, entende-se que essas são apenas algumas das possibilidades de manifestação dentro do espectro das intensidades da AF (leve, moderada e vigorosa). Quanto às inferências, assume-se a possibilidade de existência da causalidade reversa da associação dos comportamentos investigados com o IMC, tendo em vista o delineamento transversal do estudo. Ou seja, ao assumir essa limitação os resultados precisam de cautela na interpretação, visto que alterações no IMC podem levar a mudança dos níveis de AF, tempo de televisão e consumo de AUPs.

Por outro lado, as análises incluíram dados representativos de 10.926 adolescentes da rede pública e privada de todo o país, fornecendo dados com reduzido viés de contextos socioculturais por considerar a discriminação das regiões do país (Norte, Nordeste, Centro-Oeste, Sudeste e Sul) e características da escola (pública e privada). Cabe ressaltar o emprego das análises de regressão quantílica, de caráter inovador na investigação de comportamentos do estilo de vida de adolescentes. Ainda, a forma de coleta do questionário apresentar interface mais amigável com os escolares e aproveitar da usabilidade dos celulares nessa população oferece poucas perdas no autorrelato ${ }^{22}$.

\section{Conclusões}

O presente estudo indica que a associação entretempo excessivo frente à TV e IMC ocorreu nos 
diferentes percentis do IMC, independente da AF e é potencializado pelo consumo diário de AUPs em adolescentes. Dessa maneira, sugere-se a adoção de políticas públicas que estimulem a prática de AF nos mais distintos contextos: na escola, em casa e na comunidade; que difundam a compreensão de que tempo excessivo frente à TV em crianças e adolescentes contribui para a obesidade e que implementem políticas regulatórias de proibição de propaganda de AUPs para essa faixa etária, de taxação a AUPs e de subsídios aos alimentos saudáveis, como frutas e legumes ${ }^{28,29}$.

\section{Colaboradores}

CLM Lourenço e M Christofoletti participaram de todas as fases do estudo, desde a concepção e planejamento, análise e interpretação dos dados até a redação do rascunho e aprovação da versão final do manuscrito. EL Mendes e DC Malta contribuíram substancialmente para a interpretação dos dados e revisão crítica do conteúdo e aprovação da versão final do manuscrito. 


\section{Referências}

1. Ng M, Fleming T, Robinson M, Thomson B, Graetz N, Margono C, Mullany EC, Biryukov S, Abbafati C, Abera SF, Abraham JP, Abu-Rmeileh NME, Achoki T, AlBuhairan FS, Alemu ZA, Alfonso R, Ali MK, Ali R, Guzman NA, Ammar W, Anwari P, Banerjee A, Barquera S, Basu S, Bennett DA, Bhutta Z, Blore J, Cabral N, Nonato IC, Chang J-C, Chowdhury R, Courville KJ, Criqui MH, Cundiff DK, Dabhadkar KC, Dandona L, Davis A, Dayama A, Dharmaratne SD, Ding EL, Durrani AM, Esteghamati A, Farzadfar F, Fay DFJ, Feigin VL, Flaxman A, Forouzanfar MH, Goto A, Green MA, Gupta R, Hafezi-Nejad N, Hankey GJ, Harewood HC, Havmoeller R, Hay S, Hernandez L, Husseini A, Idrisov BT, Ikeda N, Islami F, Jahangir E, Jassal SK, Jee SH, Jeffreys M, Jonas JB, Kabagambe EK, Khalifa SEAH, Kengne AP, Khader YS, Khang Y-H, Kim D, Kimokoti RW, Kinge JM, Kokubo Y, Kosen S, Kwan G, Lai T, Leinsalu M, Li Y, Liang X, Liu S, Logroscino G, Lotufo PA, Lu Y, Ma J, Mainoo NK, Mensah GA, Merriman TR, Mokdad AH, Moschandreas J, Naghavi M, Naheed A, Nand D, Narayan KMV, Nelson EL, Neuhouser ML, Nisar MI, Ohkubo T, Oti SO, Pedroza A, Prabhakaran D, Roy N, Sampson U, Seo H, Sepanlou SG, Shibuya K, Shiri R, Shiue I, Singh GM, Singh JA, Skirbekk V, Stapelberg NJC, Sturua L, Sykes BL, Tobias M, Tran BX, Trasande L, Toyoshima H, van de Vijver S, Vasankari TJ, Veerman JL, Velasquez-Melendez G, Vlassov VV, Vollset SE, Vos T, Wang C, Wang XR, Weiderpass E, Werdecker A, Wright JL, Yang YC, Yatsuya H, Yoon J, Yoon S-J, Zhao Y, Zhou M, Zhu S, Lopez AD, Murray CJL, Gakidou E. Global, regional, and national prevalence of overweight and obesity in children and adults during 1980-2013: a systematic analysis for the Global Burden of Disease Study 2013. Lancet 2014; 384(9945):766-781.

2. Rivera JÁ, Cossío TG, Pedraza LS, Aburto TC, Sánchez TG, Martorell R. Childhood and adolescent overweight and obesity in Latin America: a systematic review. Lancet Diabetes Endocrinol 2014; 2(4):321-332.

3. Brasil. Ministério do Planejamento, Orçamento e Gestão. Pesquisa de Orçamentos Familiares (POF) 2008-2009. Rio de Janeiro: IBGE; 2010.

4. Harrington DM, Staiano AE, Broyles ST, Gupta AK, Katzmarzyk PT. BMI percentiles for the identification of abdominal obesity and metabolic risk in children and adolescents: Evidence in support of the CDC 95th percentile. Eur J Clin Nutr 2013; 67(2):218-222.

5. Kumar S, Kelly AS. Review of Childhood Obesity: From Epidemiology, Etiology, and Comorbidities to Clinical Assessment and Treatment. Mayo Clin Proc 2017; 92(2):251-265.

6. Pereira-Lancha LO, Campos-Ferraz PL, Lancha AH. Obesity: considerations about etiology, metabolism, and the use of experimental models. Diabetes Metab Syndr Obes 2012; 5:75-87.

7. Enes CC, Slater B. Obesity in adolescence and its main determinants. Rev Bras Epidemiol 2010; 13(1):163-171

8. Tremblay MS, Aubert S, Barnes JD, Saunders TJ, Carson V, Latimer-Cheung AE, Chastin SFM, Altenburg TM, Chinapaw MJM, SBRN Terminology Consensus Project Participants. Sedentary Behavior Research Network (SBRN) - Terminology Consensus Project process and outcome. Int J Behav Nutr Phys Act 2017; 14:75.
9. American Academy of Pediatrics. Policy Statement Children, Adolescents, Obesity, and the Media. Pediatrics 2011; 128(1):201-208.

10. Sociedade Brasileira de Pediatria (SBP). Saúde de Crianças e Adolescentes na Era Digital. Departamento de Adolescência, editor. Manual de Orientação [Internet]. 2016 [acessado 13 out 2018]. Disponível em: https://www.sbp.com.br/fileadmin/user_upload/2016/11/19166d-MOrient-Saude-Crian-e-Adolesc pdf.

11. Guerra PH, Ribeiro EHC, Leme ACB, Mota J, Farias Júnior JC, Florindo AA. Sedentary behavior and body composition in children of low-and mid-income countries: a review. Rev Bras Ativ Fis Saude 2018; 23:1-11.

12. Zhang J, Seo D-C, Kolbe L, Middlestadt S, Zhao W Associated Trends in Sedentary Behavior and BMI Among Chinese School Children and Adolescents in Seven Diverse Chinese Provinces. Int J Behav Med 2012; 19(3):342-350.

13. Petribú MMV, Tassitano RM, Nascimento WMF, Santos EMC, Cabral PC. Factors associated with overweight and obesity among public high school students of the city of Caruaru, Northeast Brazil. Rev Paul Pediatr 2011; 29(4):536-545.

14. Beyerlein A. Quantile Regression-Opportunities and Challenges From a User's Perspective. Am J Epidemiol 2014; 180(3):330-331.

15. Yu K, Lu Z, Stander J. Quantile regression: applications and current research areas. J R Stat Soc Ser Stat 2003; 52(3):331-350.

16. Mitchell JA, Dowda M, Pate RR, Kordas K, Froberg K, Sardinha LB, Kolle E, Page A. Physical Activity and Pediatric Obesity: A Quantile Regression Analysis. Med Sci Sports Exerc 2017; 49(3):466-473.

17. Pearson N, Biddle SJH. Sedentary Behavior and Dietary Intake in Children, Adolescents, and Adults: A Systematic Review. Am J Prev Med 2011; 41(2):178188.

18. Costa CS, Flores TR, Wendt A, Neves RG, Assunção MCF, Santos IS. Comportamento sedentário e consumo de alimentos ultraprocessados entre adolescentes brasileiros: Pesquisa Nacional de Saúde do Escolar (PeNSE), 2015. Cad Saude Publica 2018; 34:e00021017.

19. Monteiro CA, Cannon G, Moubarac J-C, Levy RB, Louzada MLC, Jaime PC. The UN Decade of $\mathrm{Nu}-$ trition, the NOVA food classification and the trouble with ultra-processing. Public Health Nutr 2018; 21(1):5-17.

20. Louzada MLC, Baraldi LG, Steele EM, Martins APB, Canella DS, Moubarac J-C, Levy RB, Cannon G, Afshin A, Imamura F, Mozaffarian D, Monteiro CA. Consumption of ultra-processed foods and obesity in Brazilian adolescents and adults. Prev Med 2015; 81:9-15.

21. Tavares LF, Fonseca SC, Garcia Rosa ML, Yokoo EM Relationship between ultra-processed foods and metabolic syndrome in adolescents from a Brazilian Family Doctor Program. Public Health Nutr 2012 15(1):82-87.

22. Instituto Brasileiro de Geografia e Estatística (IBGE). Pesquisa Nacional de Saúde do Escolar: 2015. Rio de Janeiro: IBGE; 2016. 
23. World Health Organization (WHO). Global School-based Student Health survey (GSHS) [Internet]. [acessado 13 out 2018]. Disponível em: https://www. who.int/ncds/surveillance/gshs/en/.

24. Oliveira MM, Campos MO, Andreazzi MAR, Malta DC. Características da Pesquisa Nacional de Saúde do Escolar - PeNSE. Epidemiol Serv Saude 2017; 26(3):605-616.

25. Physical Activity Guidelines Advisory Committee. Physical Activity Guidelines Advisory Committee Report, 2008. Washington, D.C.: Department of Health and Human Services; 2008.

26. Lourenço CLM, Zanetti HR, Amorim PRS, Mota JAPS, Mendes EL. Comportamento sedentário em adolescentes: prevalência e fatores associados. Rev Bras Cien Mov 2018; 26(3):23-32.

27. Regis MF, Oliveira LMFT, Santos ARM, Leonidio ACR, Diniz PRB, Freitas CMSM. Urban versus rural lifestyle in adolescents: associations between environment, physical activity levels and sedentary behavior. Einstein 2016; 14(4):461-467.

28. Sousa JG, Lima LR, Fernandes CRS, Santos GM. Atividade física e hábitos alimentares de adolescentes escolares: Pesquisa Nacional de Saúde do Escolar (PENSE), 2015. Rev Bras Nutr Esport 2019; 13(77):87-93.

29. Chaves VLV, Freese E, Lapa TM, Cesse EÂP, Vasconcelos ALR. Evolução espaço-temporal do sobrepeso e da obesidade em adolescentes masculinos Brasileiros, 1980 a 2005. Cad Saude Publica 2010; 26:1303-1313.

30. Evensen E, Wilsgaard T, Furberg A-S, Skeie G. Tracking of overweight and obesity from early childhood to adolescencein a population-based cohort - the Tromsø Study, Fit Futures. BMC Pediatr 2016; 16:64.

31. Hancox RJ, Milne BJ, Poulton R. Association between child and adolescent television viewing and adult health: a longitudinal birth cohort study. Lancet 2004; 364(9430):257-262.
32. Maia EG, Costa BVL, Coelho FS, Guimarães JS, Fortaleza RG, Claro RM. Análise da publicidade televisiva de alimentos no contexto das recomendações do Guia Alimentar para a População Brasileira. Cad Saude Publica 2017; 33(4):e00209115.

33. Martins APB, Levy RB, Claro RM, Moubarac JC, Monteiro CA. Participação crescente de produtos ultraprocessados na dieta brasileira (1987-2009). Rev Saude Publica 2013; 47(4):656-665.

34. Leech RM, McNaughton SA, Timperio A. The clustering of diet, physical activity and sedentary behavior in children and adolescents: a review. Int J Behav Nutr Phys Act 2014; $11: 4$.

35. Lourenço CLM, Silva Filho RCS, Hauser E, Barbosa AR, Mendes EL. Cluster and simultaneity of modifiable risk factors for cardiovascular diseases in adolescents of Southeast Brazil. Mot Rev Educ Fis 2020; 26(2):e10200033.

36. Finkelstein EA, Graham WCK, Malhotra R. Lifetime Direct Medical Costs of Childhood Obesity. Pediatrics 2014; 133(5):854-862.

37. Apovian CM. Obesity: definition, comorbidities, causes, and burden. Am J Manag Care 2016; 22(Supl. 7):s176-s185.

Artigo apresentado em 29/04/2020

Aprovado em 30/09/2020

Versão final apresentada em 02/10/2020

Editores-chefes: Romeu Gomes, Antônio Augusto Moura da Silva 